\title{
Determinación de la betalactamasa en cepas de Neisseria gonorrhoeae
}

\author{
Vela M.C. ${ }^{1}$, de Vargas C.I. ${ }^{1}$, Castañeda E. ${ }^{1}$
}

\section{RESUMEN}

La aparición de cepas de $N$. gonorrhoeae resistentes a diferentes antibióticos, la cual puede estar mediada por plásmidos o por mutaciones, requiere una continua vigilancia, con el fin de revisar los esquemas de tratamiento. En nuestro medio, los estudios realizados para establecer el problema de resistencia de cepas de $N$. gonorrhoeae a la penicilina han sido escasos, por esto decidimos determinar, en aislamientos de este patógeno obtenidos en diferentes servicios de salud del país, la presencia de la betalactamasa y la susceptibilidad antimicrobiana.

De 89 cepas estudiadas, 46 (52\%) fueron productoras de beta lactamasa (PBL); 11\% de ellas presentaron resistencia a la espectinomicina y $96 \%$ a la tetraciclina. En las cepas no productoras de beta lactamasa, el $61 \%$ fue resistente a la tetraciclina y el $2 \%$ a la penicilina; adicionalmente el $98 \%$ de las cepas NPBL presentaron una sensibilidad moderada a la penicilina. Todas las cepas, PBL y NPBL, fueron sensibles a la ceftriaxona. Estos datos confirman la circulación de cepas de N. gonorrhoeae PBL en diferentes regiones del país y apoyan la recomendación de mantener una vigilancia que nos permita conocer la verdadera frecuencia de ellas en nuestro medio.

\section{SUMMARY}

The appearance of strains of $N$.gonorrhoeae resistant to different antibiotics, which could be caused by plasmids or by mutations, require constant surveillance so as to revise the schemes of treatment. In our country the existent studies to determine the problem of strains of $N$. gonorrhoeae resistent to penicilin have been scarce therefore it was decided to determine the presence of beta lactamase and antimicrobe susceptibility from isolates of this pathogen obtained from different health services around the country.

From 89 strains studied 46 (52\%) were producers of beta lactamase; $11 \%$ of these presented resistance to spectinomycin and $96 \%$ to tetracycline. In the strains which did not produce beta lactamase, $61 \%$ were resistant to tetracycline and $2 \%$ to penicilin: additionally $98 \%$ of the NPBL strains presented a moderate sensitivity to penicilin. All the PBL and NPBL strains were sensitive to ceftriaxone. This data confirms the circulation of PBL strains of $N$. gonorrhoeae throughout different regions of the country and supports the recommendation to maintain surveillance, permitting the determination of the real frequency of these strains in our country.

La bacteria que se conoce actualmente como Neisseria gonorrhoeae fue descrita por Albert

1 Grupo de Microbiología, Instituto Nacional de Salud
Neisser en el año de 1879, en extendidos de secreciones urogenitales y oculares; posteriormente, en 1885, Bumm confirmó que constituía un patógeno para el hombre $(1,2)$. La $N$. 
gonorrhoeae es un diplococo Gram negativo, que requiere para su crecimiento medios suplementados con aminoácidos, factor $\mathrm{X}$ (hemina) suministrado por la hemoglobina, factor $\mathrm{V}$ (nicotinamida-adenin-dinucleótido) (NAD), una atmósfera microaerofílica y una temperatura de $35^{\circ} \mathrm{C}$; posee la indofenol oxidasa y tiene la capacidad de fermentar la glucosa $(3,4)$.

Los estudios sobre la patogénesis de la $N$. gonorrhoeae han demostrado que ataca el epitelio columnar no ciliado presente en las mucosas, causando inflamación y secreción purulenta del aparato genitourinario de hombres y de mujeres, de la faringe, del recto y de la conjuntiva. También se disemina por vía hematógena y puede afectar principalmente las articulaciones y meninges $(4,5)$.

La terapia para las infecciones causadas por $N$ gonorrhoeae se inició en 1935 con las sulfonamidas, en 1943 se empleó la penicilina, con dosis de 50.000 UI a 100.000 UI a la cual eran sensibles el $99 \%$ de los aislamientos. Desde el inicio de la terapia con penicilina, se ha observado una tendencia del gonococo a la resistencia, por esta razón se ha incrementado la dosis; es así como en 1972 la dosis recomendada fue de 4,5 millones UI más 1 gramo de probenecid y en la actualidad es de 4,8 millones de UI $(4,6)$.

En 1976 se describieron en Estados Unidos las primeras cepas productoras de beta lactamasa (PBL), mediada su producción por un plásmido, el cual, de acuerdo a estudios epidemiológicos tuvo su origen en Asia $(4,7)$. Entre 1980 y 1982 la incidencia de cepas PBL se incrementó alcanzando en ese último año un total de 4.500 casos $(4,5,8,9)$. En 1984 se detectaron cepas resistentes a penicilina pero no productoras de beta lactamasa; se determinó que esta resistencia era cromosomal $(4,10)$.

Muchas cepas PBL son también resistentes a la tetraciclina, y esta resistencia es mediada por el cromosoma o por plásmidos $(4,11)$; actualmente sehan descrito cepas resistentes a espectinomicina mediada la resistencia por el cromosoma $(4,12)$.

Los estudios realizados en nuestro medio con el fin de establecer el problema de la resistencia de las cepas de N. gonorrhoeae a la penicilina han sido muy escasos. En un estudio inicial realizado en Medellín en 1977, se determinó la concentración inhibitoria mínima (CIM) de la penicilina G en 41 cepas de $N$. gonorroheae y se encontró, en el $5 \%$ de ellas, una $\mathrm{CIM}>2,0 \mathrm{U} / \mathrm{ml}$ por lo cual fueron consideradas como resistentes (13). En 1986, en Cali, se informaron las primeras cepas PBL aisladas en 1 de 22 hombres y en 2 de 46 mujeres (14); en 1992, en otro estudio realizado en Medellín, en pacientes que presentaron fallas en el tratamiento, se determinaron 54 de 126 (43\%) cepas PBL (15).

Con base en los anteriores datos se quiso establecer la presencia de la enzima beta lactamasa en cepas aisladas en diferentes regiones del país, las cuales son enviadas al Instituto Nacional de Salud (INS) como parte de un programa de red de laboratorios. Se estudiaron 89 cepas y se determinó la presencia de la beta lactamasa, por los tres métodos estandarizados, en 46 de ellas (52\%). En el $96 \%$ de las cepas PBL se determinó resistencia a la tetraciclina y en el $11 \%$ a la espectinomicina; en el $98 \%$ de las cepas no productoras de beta lactamasa (NPBL) se determinó una sensibilidad moderada a la penicilina. Todas las cepas estudiadas fueron sensibles a la ceftriaxona.

\section{Materiales y métodos}

\section{Cepas}

Se estudiaron 89 cepas de N. gonorrhoeae enviadas por diferentes servicios de salud e identificadas con los siguientes datos: procedencia, sexo y edad del paciente y pruebas de laboratorio realizadas para su identificación.

Cuarenta y una de las cepas habían sido recibidas de 1983 a 1991 y 48 cepas fueron recuperadas de 109 enviadas de 1992 a 1993.

Como cepas control se utilizaron dos cepas de Staphylococcus aureus (ATCC 29213 y ATCC 25923) productora y no de beta lactamasa y una cepa de N. gonorrhoeae (INS 008) no productora de beta lactamasa.

\section{Confirmación de cepas}

Las cepas fueron recibidas en agar Thayer Martin (microcultivo INS) y se resembraron inmediata- 
mente en cajas con agar chocolate con hemoglobina bovina (BBL) al $1 \%$ y suplemento (Isovitalex $\mathrm{BBL}$ ) al $1 \%$. Las cajas se incubaron a $35^{\circ} \mathrm{C}$ por 24-72 horas en una atmósfera de $5 \%$ de $\mathrm{CO} 2$ y una humedad del $90 \%(16,17)$. Con las colonias compatibles se realizó una coloración de Gram y se confirmó su identificación con las pruebas de oxidasa y fermentación de azúcares $(3,16,17)$.

\section{Determinación de la beta lactamasa}

Se utilizaron los tres métodos estandarizados. Para el método cromogénico se emplearon discos de cefinasa $(B B L)(4,16,18)$; para el método acidométrico se empleó como substrato una solución de $10 \times 10^{6} \mathrm{UI}$ de penicilina $\mathrm{G}$ y rojo de fenol como indicador $(4,16,18)$, y para el método yodométrico se empleó una mezcla de almidónyodo y $10.000 \mathrm{UI}$ de penicilina $\mathrm{G}(4,16,18)$.

\section{Susceptibilidad antimicrobiana}

Se determinó por el método de difusión en agar de Kirby Bauer, empleando el medio agar GC (BBL) con suplemento al $1 \%$ (Isovitalex-BBL, Vitox-Oxoid) según las recomendaciones del Comité Nacional de Estándares para los Laboratorios Clínicos (NCCLS) $(6,19)$. Se utilizaron discos de penicilina de $10 \mathrm{UI}$ (Difco), ceftriaxona 30 mcg (Difco), tetraciclina $30 \mathrm{mcg}$ (Difco) y espectinomicina $100 \mathrm{mcg}$; estos últimos fueron preparados en el laboratorio. La lectura e interpretación de los datos se hizo de acuerdo con las instrucciones del comité (NCCLS) (19).

\section{Resultados}

\section{Cepas}

De 1983 a 1991 se habían recibido y confirmado 41 cepas y entre febrero de 1992 a abril de 1993 se recibieron 109 , de las cuales se recuperaron $48(44 \%)$. La viabilidad de las cepas estuvo relacionada con el tiempo transcurrido entre su envío y la recepción en el laboratorio del INS. Se recuperaron $44 / 72(61 \%)$ de las cepas recibidas en las 72 horas siguientes al envío y $4 / 37$ (11\%) de las recibidas entre los 4 y 7 días.

La procedencia de las 89 cepas fue la siguiente: 4 de Antioquia, 1 de Amazonas, 15 de Atlántico, 5 de Boyacá, 6 de Caldas, 3 de Guaviare, 4 de
Norte de Santander, 1 de Quindío, 1 de Risaralda y 49 de Santafé de Bogotá.

En relación a la distribución de las cepas por sexo, grupo de edad y origen de la muestra anotamos, en el sexo femenino $55(62 \%)$ del cérvix de mujeres adultas y $11(12 \%)$ de niñas menores de 8 años de edad: 2 (18\%) de conjuntiva y $9(82 \%)$ de vagina. En el sexo masculino, $18(20 \%)$ de uretra de hombres adultos y $5(6 \%)$ de menores de ocho años: $4(80 \%)$ de conjuntiva y $1(20 \%)$ de uretra.

\section{Confirmación de las cepas}

Las 89 cepas fueron confirmadas como $N$. gonorrhoeae.

\section{Determinación de la beta lactamasa}

Por los métodos acidométrico y cromogénico se determinó que 46 de 89 (52\%) cepas eran productoras de la enzima, pero la sensibilidad inicial del método yodométrico fue del $15 \%(7 / 46)$; al realizarse por segunda vez este método se obtuvo una sensibilidad del $100 \%$.

En la tabla 1, se observa el número de cepas enviadas por los diferentes servicios de salud y el número y porcentaje de cepas PBL en cada uno de ellos. En la figura 1, se presenta la distribución de las cepas PBL y NPBL por año de aislamiento.

Tabla 1. Procedencia de las cepas productoras de beta lactamasa.

\begin{tabular}{lcrr}
\hline Procedencia & $\begin{array}{c}\text { Total cepas } \\
\text { confirmadas }\end{array}$ & n (\%) & PBL* \\
\hline Antioquia & 4 & 2 & $(50)$ \\
Amazonas & 1 & 0 & $(0)$ \\
Atlántico & 15 & 9 & $(60)$ \\
Boyacá & 5 & 5 & $(100)$ \\
Caldas & 6 & 5 & $(83)$ \\
Guaviare & 3 & 3 & $(100)$ \\
Norte de Santander & 4 & 3 & $(75)$ \\
Quindio & 1 & 0 & $(0)$ \\
Risaralda & 1 & 1 & $(100)$ \\
Santafé de Bogotá & 49 & 18 & $(37)$ \\
\hline TOTAL & 89 & & \\
\hline
\end{tabular}

*PBL $=$ productoras de beta lactamasa 


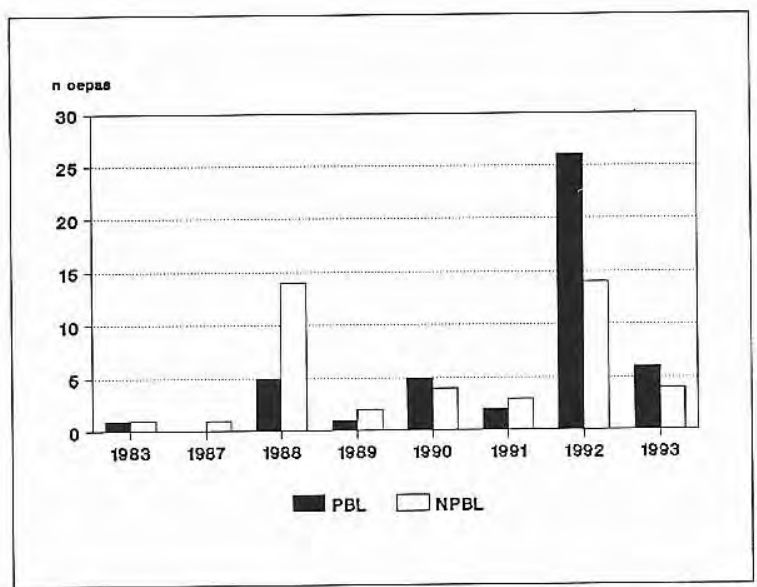

Figura 1. Número de cepas de Neisseria gonorrhoeae productoras y no productoras de beta lactamasa por año de aislamiento.

En relación a la distribución de las cepas $\mathrm{PBL}$ por sexo, encontramos que $33 / 55(62 \%)$ eran de mujeres adultas, $5 / 11$ (45\%) de niñas, $6 / 18(33 \%)$ eran de hombres adultos y $2 / 5(40 \%)$ eran de niños.

\section{Susceptibilidad antimicrobiana}

El análisis de los resultados de la susceptibilidad antimicrobiana se realizó por separado en las cepas PBL y en las NPBL. En la figura 2 encontramos los resultados obtenidos con las cepas PBL; todas fueron sensibles a la ceftriaxona, el $11 \%$ fueron resistentes a la espectinomicina, el $96 \%$ fueron resistentes a la tetraciclina y todas fueron resistentes a la penicilina. Las cepas resistentes a la espectinomicina fueron todas enviadas por el Servicio de Salud de Santafé de Bogotá.

En la figura 3 observamos el comportamiento de las cepas NPBL con los diferentes antibióticos, todas las cepas fueron sensibles a la ceftriaxona y a la espectinomicina, $61 \%$ fueron resistentes a tetraciclina y $98 \%$ moderadamente sensibles a la penicilina.

\section{Discusión}

La baja viabilidad de las cepas confirma lo descrito en la literatura, con referencia a la gran sensibilidad de las especies patógenas del género Neisseria a los cambios de temperatura y

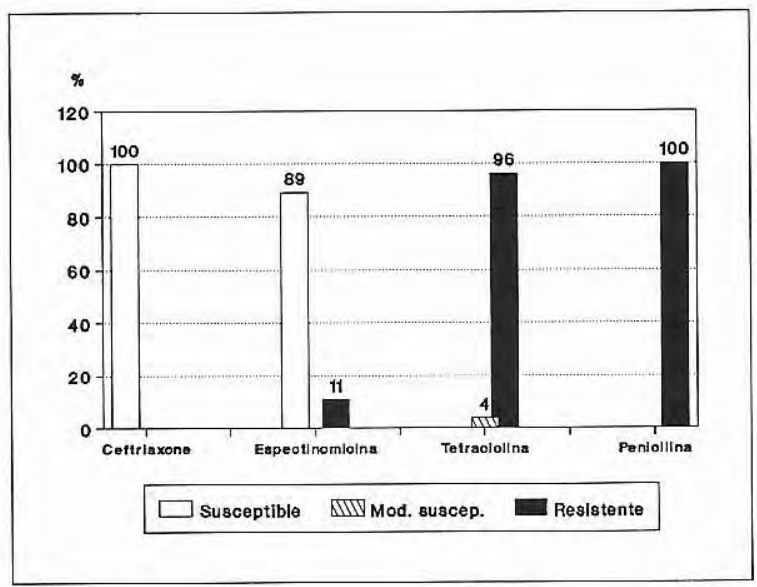

Figura 2. Sensibilidad de lascepas de Neisseria gonorrhoeae productoras de beta lactamasa.

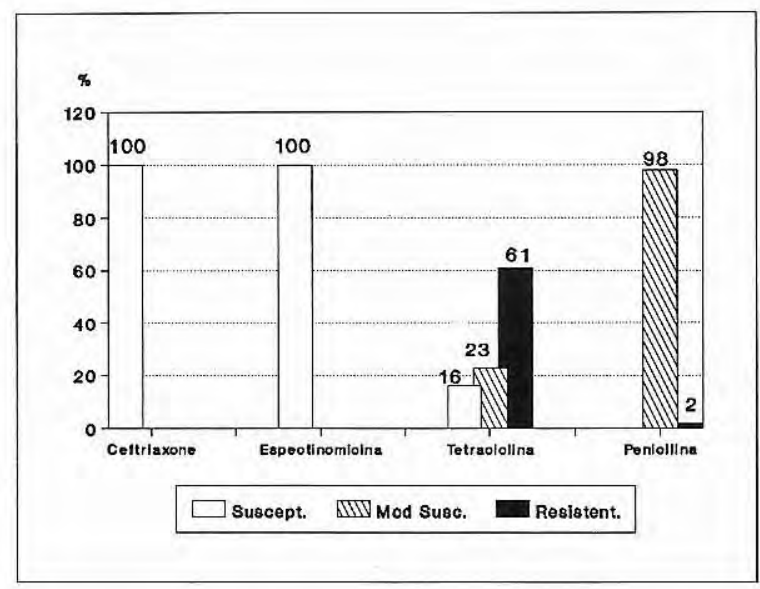

Figura 3. Sensibilidad de las cepas de Neisseria gonorrhoeae no productoras de beta lactamasa.

humedad, debido en parte a la activación de algunas enzimas autolíticas (3). De tal manera que el medio de transporte y el tiempo requerido para el envío son factores críticos cuando se trabaja con estos patógenos $(1,4,16)$.

Es importante señalar que no se conocía la historia clínica de los pacientes debido a que las cepas fueron remitidas al INS por los laboratorios de los servicios de salud, como parte del programa de confirmación de aislamientos. Por lo tanto, no se pudo determinar si la población estudiada era de alto riesgo o población general. 
El obtener cepas de sólo 10 de los 33 servicios nos señala el problema de la no realización del cultivo para el diagnóstico de la infección gonocócica; esta recomendación se ha formulado especialmente para el diagnóstico en la mujer, debido a que la sensibilidad del examen directo coloreado con Gram es del $45 \%$ al $65 \%$ $(3,4)$; adicionalmente, es necesario realizar el cultivo en todas las muestras de menores de edad debido a la necesidad de realizar una identificación completa del microorganismo por las implicaciones médico-legales (3).

La confirmación de todas las cepas enviadas señala que a pesar de ser la N. gonorrhoeae un microorganismo fastidioso, su recuperación en el medio selectivo y su identificación, son posibles en un laboratorio de bacteriología de mediana complejidad. A partir del crecimiento en el medio selectivo, la coloración de Gram y la determinación de la oxidasa permiten establecer un diagnóstico presuntivo $(2,3)$. Esta consideración no es válida cuando se procesan las muestras extragenitales o de niños, las cuales requieren estudios confirmatorios $(1,3)$.

La procedencia de las cepas $\mathrm{PBL}$ indica que están circulando en otras regiones del país, diferentes de Cali y Medellín donde habían sido descritas inicialmente $(14,15)$.

El hallazgo señala también la importancia de establecer una vigilancia epidemiológica con los aislamientos, recomendación que fue formulada cuando aparecieron las primeras cepas resistentes y que es llevada a cabo con éxito en los países desarrollados $(4,10)$.

Al evaluar los tres métodos estandarizados para la determinación de la beta lactamasa, encontramos que el método acidométrico tiene un bajo costo y sus reactivos son estables a bajas temperaturas $\left(-20^{\circ} \mathrm{C}\right)$; el método cromogénico es más costoso pero se obtiene comercialmente, además es fácil y rápido de realizar; el método yodométrico presentó problemas debido a las dificultades para estandarizar el inóculo y a la inestabilidad de los reactivos.

La sensibilidad de todas las cepas a la ceftriaxona permite considerarla como una excelente alternativa para el tratamiento, lo cual está de acuer- do con las recomendaciones del CDC de Atlanta $(5,18)$; este fármaco no debe ser usado en infecciones faríngeas (4).

La resistencia de la $N$. gonorrhoeae a la espectinomicina ha sido informada desde 1981 en cepas PBL y NPBL; en 1985, en Estados Unidos, el $5 \%$ de las cepas aisladas fueron resistentes (12). Zenilman y colaboradores sugirieron que la resistencia a la espectinomicina aparece generalmente cuando ya existe resistencia cromosomal a otros antibióticos y que debe realizarse una investigación epidemiológica en estos casos (12). Dallabeta considera que debido a la baja frecuencia de cepas resistentes a la espectinomicina, este antibiótico puede utilizarse en el tratamiento para las infecciones gonocócicas no complicadas (4). Las cepas de este estudio que fueron resistentes a la espectinomicina, eran PBL.

La alta resistencia a la tetraciclina de las cepas PBL y NPBL revela la poca utilidad de la droga en el tratamiento de las infecciones gonocócicas (11).

La resistencia de las cepas NPBL a la penicilina es cromosomal; esta resistencia fue descrita desde 1983 en Estados Unidos (4). Debido a que estas cepas no pueden ser detectadas por la prueba de la beta lactamasa, se recomienda realizar la susceptibilidad antimicrobiana por el método de Kirby Bauer, cuando falla el tratamiento o en zonas donde la frecuencia de estas cepas sea alta $(4,6)$. Por lo general, las cepas con resistencia cromosomal son más frecuentes en las zonas donde es posible la automedicación (4).

Cuando se realizó este estudio, no se encontraron datos sobre la resistencia a la tetraciclina o a la espectinomicina de cepas de $N$. gonorrhoeae aisladas en Colombia; por lo tanto es importante continuar con esta determinación para conocer la frecuencia real de estas cepas en las diferentes regiones del país.

Los datos obtenidos en este trabajo nos obligan a recomendar a todos los laboratorios la determinación de la enzima beta lactamasa en todos los aislamientos de N. gonorrhoeae aisladas. Por lo tanto y como elemento de vigilancia epidemio- 
lógica, se debe realizar cultivo a todas las muestras cervicales, a las muestras provenientes de menores de edad y a un porcentaje de las uretrales, utilizando el medio selectivo de Thayer Martin.

\section{Agradecimientos}

A Gloria Inés Uribe, Gloria Patricia González, Mabel Merino, Luz Mary Forero, María Elsy Martínez, Gladys Mora, Luz Emilia Villegas y Marta Lucía Villa, bacteriólogas de los servicios de salud que remitieron las cepas para este estudio.

\section{Referencias}

1. Ehret JM, Judson FN, Biddle JW. Gonorrhoeae. In: Laboratory methods for the diagnosis of sexually trasnmitted diseases. BB Wentworth, FN Judson Washington, D.C. American Public Health Assosiation. 1987:4379.

2. Morse SA. Neisseria and Branhamella. Infectiuns Disease and Medical Microbiology. Second Edition. Al Braude, CE Davis, J Fierrer.Philadelphia: WB Saunders' Company . USA; 1986: 278-284.

3. Evangelista AT, Beilstein. Laboratory Diagnosis of Gonorrhoea. Cumitech 4A. Washington, D.C. USA. American Society for Mycrobiology 1993.

4. Dallabeta G, Hook III EW. Gonococcal infections. Infect Dis Clin North Amer 1987;1:25.

5. Centers for Disease Control: Antibiotic resistant strains of Neisseria gonorrhoeae: Policy guidelines for detection, management and control. MMWR 1987; 36.

6. Neumann M, Sahm D, Thornssberry C, Mc Gowan J. New developments in antimicrobial agent susceptibility testing: a practical guide. Cumitech $6 \mathrm{~A}$. Washington, D.C. USA American Society for Microbiology 1991.

7. Roberts M, Elwell L, Falkon S. Introduction to the mechanisms of genetic exchange in the gonococcus: plasmids and conjugation in Neisseria gonorrhoeae.
Immunology of Neisseria gonorrhoeae. Washington, D.C.American Society for Microbiology 1978.

8. Phillps I. Beta lactamase-producing, penicillin resistant gonococcus. Lancet 1976; 2:656.

9. Ashford WA, Goiash RG., Hemming VG. Penicillinaseproducing Neisseria gonorrhoeae. Lancet 1976; 2:657.

10. Rice RJ, Biddle JW, Jeanlouis Y,et al. Chromo-somally mediated resistance in Neisseria gonorrhoeae in the United States: results of surveillance and reporting, 1983 - 1984. J Infect Dis 1986; 153:340.

11. Sarafin SK, Genco CA, Roberts MC, Knapp J. Acquisition of beta lactamasa and TetM - containing conjugative plasmids by phenotypically diferent strains of Neisseria gonorrhoeae. Sex Transm Dis 1990; 17:67.

12. Zenilman JM, Nims LJ, Nims L, et al. Spectinomycinresistant gonococcal infections in the United States, 1985-1986. J Infect Dis 1987;156: 1002.

13. Urbina D, Montoya F. Determinación de la concentración inhibitoria mínima (CIM) de la penicilina G requerida por cepas de Neisseria gonorrhoeae aisladas en la ciudad de Medellín. Acta Med Colomb 1978;3:1.

14. Arango C, Bergonzoli G, Zafra G, Sarria MN. Prevalencia de la Neisseria gonorrhoeae productora de beta lactamasa. Colomb Med 1986;17:70.

15. Restrepo M, Gómez MV. Neisseria gonorrhoeae productora de beta lactamasa. Acta Med Colomb 1992;17:22.

16. Morello JA, Janda WM, Doern GV. Neisseria and Brahamella. Manual of Clinical Microbiology. Fith Edition. A Balows, WJ Hausler, Hd Isemberg, HJ Shadomy, KL Herrmann (Eds). Washington, D.C. American Society for Microbiology 1991: 258-276.

17. Guzman M, Vargas Cl. Neisseria. En: Microbiología Médica. Manual de procedimientos. Serie de Publicaciones Científicas No 14. Bogotá Instituto Nacional de Salud. 1988:131-148.

18. Centers for Disease Control: Procedures for use by the laboratory in the isolation and identification of Neisseria gonorrhoeae. Atlanta, Georgia; 1987:27-30.

19. Jones RN, Barry AL, Gavan TL, et al. Performance standards for antimicrobial disk susceptibility test NCCLS. Third edition. $1984 ; 4(16)$. 